University of Montana

ScholarWorks at University of Montana

2006

\title{
A Practical Look at the Variable Area Transect
}

Solomon Z. Dobrowski

University of Montana - Missoula

Shannon K. Murphy

Follow this and additional works at: https://scholarworks.umt.edu/forest_pubs

Part of the Forest Management Commons

Let us know how access to this document benefits you.

\section{Recommended Citation}

Dobrowski, Solomon Z. and Murphy, Shannon K., "A Practical Look at the Variable Area Transect" (2006). Forest Management Faculty Publications. 30.

https://scholarworks.umt.edu/forest_pubs/30

This Article is brought to you for free and open access by the Forest Management at ScholarWorks at University of Montana. It has been accepted for inclusion in Forest Management Faculty Publications by an authorized administrator of ScholarWorks at University of Montana. For more information, please contact scholarworks@mso.umt.edu. 


\title{
Notes
}

\section{A PRACTICAL LOOK AT THE VARIABLE AREA TRANSECT}

\author{
Solomon Z. Dobrowski ${ }^{1,3}$ and Shannon K. Murphy ${ }^{2}$ \\ ${ }^{1}$ University of California, Davis, Tahoe Environmental Research Center, One Shields Avenue, Davis, California 95616 USA \\ ${ }^{2}$ University of California, Davis, Department of Plant Pathology, One Shields Avenue, Davis, California 95616 USA
}

\begin{abstract}
The variable area transect (VAT) is a plotless density estimator that has received little attention in the ecological literature despite having potentially robust estimation properties. VAT allows for density estimations without the lengthy search times associated with other plotless density estimators. In spite of this, little has been written about the effect of varying transect widths on its density estimation properties or on the practical implementation of the VAT in field settings. An artificial population sampler was used to examine the effect of transect width on density estimates obtained using the VAT. Three transect widths were chosen corresponding to the mean object size, the largest object size, and twice the size of the largest object. Transect width had a marked effect on the quality of the density estimation, with the largest transect width resulting in significant negative biases in estimation. For the narrowest width, most estimates were within $10 \%$ of the true value for a nonrandomly distributed population. The practical considerations of choosing a VAT transect width are enumerated.
\end{abstract}

Key words: density estimation; plotless density estimator; sampling; variable area transect.

\section{INTRODUCTION}

A common task in ecological studies involves determining the density of stationary objects within plant and animal communities. Two strategies used to obtain density estimates are quadrat sampling and distance measures. Quadrats are commonly used, simple to apply, and their limitations have been well described (Kershaw 1973, Dale 1999). Namely, quadrats of different size produce different estimates of density when populations are not distributed randomly. Additionally, the degree of nonrandomness detected using quadrats depends on the size of quadrat used. This has proven to be a significant limitation in that most populations tend to be clumped or aggregated to some extent (Engeman et al. 1994).

Plotless density estimators (PDE) were developed in order to overcome the limitations of fixed-plot sampling strategies, as well as to reduce the amount of man-hours necessary for sampling. There are no fixed plots to delineate, and distance measures can be very rapid under certain circumstances. In contrast to quadrat techniques, which measure the number of organisms per unit area, PDEs attempt to estimate the mean area per organism, the inverse of density. This allows for the use of spacing

Manuscript received 22 December 2005; accepted 13 January 2006. Corresponding Editor: J. S. Brewer.

${ }^{3}$ E-mail: szdobrowski@ucdavis.edu between organisms to be used in determining mean area per organism. Consequently, density can be calculated given the mean distance between organisms (Cottam and Curtis 1956). The mean distance between organisms is determined either by measuring the distance from a random point to the $r$ th closest organism (closest individual methods) or by measuring the distance from one organism to its $r$ th closest neighbor (nearest neighbor methods). In one of the first review papers about PDEs, Cottam and Curtis (1956) compared the use of the closest individual (CI), nearest neighbor (NN), random pairs (RP), point center quarter (PCQ), and quadrat sampling techniques. They sampled three forest communities and an artificial population, and showed that for $r=1$ the CI, $\mathrm{NN}$, and RP techniques all required correction factors in order to obtain reasonable estimates of density. The PCQ did not require a correction factor and was found to be the most robust PDE they examined.

There are also a number of problems associated with PDEs. The precision and accuracy of the density estimate is dependent upon the product of the number of samples taken, $n$, and the number of measurements in each sample, $r$. Additionally, they have been shown to be sensitive to deviations from random spatial distributions (Diggle 1975, Engeman et al. 1994). In the most extensive simulation study of its type, Engeman et al. (1994) compared 24 PDEs within 96 combinations of six spatial patterns, four sample sizes, and four population 
densities. Their findings can be generalized as follows: (1) The quality of density estimations decreased as the populations deviated further from a random pattern. (2) Basic "distance" measures such as $\mathrm{CI}$ and NN were particularly sensitive to deviations from random patterns. (3) Performance improved as sample size increased (for both $n$ and $r$ ). (4) For $r=3$, ordered distance, PCQ, and the variable area transect estimators were the best overall performers. Unfortunately, using ordered distance or PCQ methods in the field with an $r$ $\geq 3$ is often impractical. This is due to the difficulty of determining which organism is the $r$ th. As $r$ increases, the time required to search the sample area for the $r$ th organism increases, and a key benefit of using a PDE (speed and efficiency) is lost.

In order to address this issue, Parker (1979) developed the variable area transect (VAT). The VAT can be considered a combination of distance and quadrat methods (Engeman et al. 1994). At each of $n$ randomly located points, a fixed-width transect is searched until the $r$ th organism is found. In this way, the $r$ th organism is encountered as a consequence of traversing the transect. Consequently, searching and measuring are accomplished at the same time. This allows for robust density estimations found with high $r$ values without the lengthy search times associated with these types of measurements.

In light of the potential advantages of the VAT, it is surprising that little work has been published using the technique. Engeman et al. (1994) showed that VAT sampling with $r=3$ was the most practical PDE considered with favorable estimation properties. In another extensive simulation study, Engeman and Sugihara (1998) showed that VAT estimation properties were most significantly improved by increasing $r$ from 3 to 4 , but leveled off above $r=6$. Most notably, there has been little written about the practical application of VAT sampling, particularly with regards to choosing appropriate values for the width of the transect. In an attempt to address this issue, Parker (1979) briefly mentioned that "difficulties might arise in determining transect boundaries" and suggested that investigators can carry a rod to determine the transect boundaries. Moreover, Engeman and Sugihara (1998) made no mention of the width of the VAT used in their simulations. The "organisms" in their simulations were dimensionless points and varied only in their location.

In contrast to these simulations, organisms in the field vary in size and shape, in addition to location. The potential applications of VAT sampling are broad, ranging from use in estimating tree density, to estimating invertebrate populations in intertidal communities such as described by Parker (1979). It is reasonable to assume that the width of the transect used should vary in relation to the size or shape of the organisms sampled. Consequently, there remains some uncertainty about the effect, if any, of transect width on the practical application of density estimation using VAT sampling.

In light of this, the scope of this paper is to introduce the reader to the use of VAT sampling. More specifically, it will address some of the practical considerations associated with choosing transect widths under certain circumstances. A sampling simulation was conducted on an artificial population in order to address these ends.

\section{Methods}

\section{Artificial population}

VAT sampling was conducted using an artificial population sampler (Schultz Developments. Walnut Creek, California, USA). The device is comprised of a square meter gradated sampling frame and a population composed of circular colored plexiglass discs varying in six size classes from $0.25 \mathrm{~cm}^{2}(0.56 \mathrm{~cm}$ diameter) to 2.5 $\mathrm{cm}^{2}$ (1.8 cm diameter). All population parameters, including density, were provided with the sampler. The population was made up of 1840 discs covering $13 \%$ of the total sample area $\left(1 \mathrm{~m}^{2}\right)$. The sampling device came with discs located with a nonrandom spatial distribution; the primary constraint being that no two discs overlapped. The mean center-to-center nearest neighbor distance was $13.62 \mathrm{~cm}$ with a standard deviation of 5.07 $\mathrm{cm}$. Using this information, the Clark and Evans (1954) aggregation index was calculated to be 1.17 . Values below 1 are considered clumped, a value of 1 is considered random, and values above 1 are considered more uniform. Further detail on the artificial population sampler can be found in Schultz et al. (1961).

\section{Sampling design}

The six size classes of the discs within the artificial population sampler provided a logical framework in which to study the effects of transect width. Three transect widths were used in the study. The first corresponded to the mean size of the six classes (1.2 $\mathrm{cm})$. The second was the width of the largest object in the population $(1.8 \mathrm{~cm})$. The third transect width was twice the size of the largest object $(3.6 \mathrm{~cm})$.

At each of the three sampling widths, 10 sample points $(n=10)$ were located in the population using pairs of random numbers taken from a random number generator. Starting at each point, the transect was searched in a random direction (i.e., the direction that provided the longest potential transect), until the fourth, fifth, and sixth object were encountered $(r=4,5,6)$, and these distances were recorded $\left(X_{i}\right)$. Objects were counted if their center fell within the transect boundary, and measurements were taken to the center of each disc along the transect. This entire procedure was replicated 20 times $(N=20)$ for each transect width configuration. This sampling design allowed us to examine nine different sampling configurations: three transect widths by three measurements in each sample $(r=4,5,6)$. 
TABLE 1. Summary of mean $(N=20)$ and variance of distance measures for different samplingwidth configurations.

\begin{tabular}{|c|c|c|c|c|c|c|}
\hline \multirow{2}{*}{$\begin{array}{l}\text { Sample width } \\
\text { (cm) }\end{array}$} & \multicolumn{3}{|c|}{ Mean $\Sigma X_{i}(\mathrm{~cm})$} & \multicolumn{3}{|c|}{ Coefficient of variation $\dagger(\%)$} \\
\hline & $r=4$ & $r=5$ & $r=6$ & $r=4$ & $r=5$ & $r=6$ \\
\hline 1.2 & 169.8 & 213.0 & 252.9 & 24.4 & 21.1 & 17.1 \\
\hline 1.8 & 104.3 & 138.9 & 169.8 & 11.5 & 20.5 & 23.8 \\
\hline 3.6 & 83.2 & 96.6 & 108.8 & 13.7 & 11.8 & 13.2 \\
\hline
\end{tabular}

Notes: Sample widths represent the mean object size $(1.2 \mathrm{~cm})$, largest object size $(1.8 \mathrm{~cm})$, and twice the largest object size $(3.6 \mathrm{~cm})$. Distance measures were recorded from a randomly located point to the $r$ th closest object within a given sample transect.

$\dagger \mathrm{cv}=\mathrm{SD} \Sigma X_{i} /$ mean $\Sigma X_{i}$.

\section{Density estimation}

Density was calculated for each sampling configuration replication $(N)$ using the formula described by Parker (1979):

$$
D=n r-1 /\left(w \sum X_{i}\right)
$$

where $n=10, w$ is the width of the VAT transect, and $\Sigma X_{i}$ is the sum of lengths searched to the $r$ th organism. The sample mean $(\bar{D})$ for $N=20$ and the relative bias (RB) was calculated using the following formula:

$$
\mathrm{RB}=(\text { estimate }- \text { true value }) / \text { true value } .
$$

\section{Resampling}

For each transect-width sampling configuration, 200 distance samples $(n \times N)$ were recorded, each containing three distance measures for $r=4,5$, and 6. From this population, $X_{i}$ was resampled with replacement 20 times $\left(n_{\mathrm{b}}=20\right)$, creating a new bootstrap sample, which was then used to calculate an estimate of density $\left(D_{\mathrm{b}}\right)$ using Eq. 1. This resampling procedure was repeated 1000 times $\left(N_{\mathrm{b}}\right)$ for each width configuration, allowing for the calculation of a bootstrapped estimate of the mean density $\left(\bar{D}_{\mathrm{b}}\right)$ and the standard error of the bootstrapped sample mean $\left(\mathrm{SE}_{\mathrm{b}}\right)$. Additionally, the relative bias of the bootstrapped sample mean $\left(\mathrm{RB}_{\mathrm{b}}\right)$ was calculated using Eq. 2. All statistics were calculated using S-Plus (Insightful 2001).

\section{RESULTS}

As would be expected, the mean distance traveled to the $r$ th organism decreased with increasing VAT width (Table 1). Variance estimates of $X_{i}$ decreased with increasing VAT width, but showed no distinct pattern with increasing $r$. Additionally, the distribution of measured $X_{i}^{\prime} s$ were normally distributed (results not shown).

Sample width showed a strong influence on density estimates (Table 2). As sample width increased beyond the largest object size $(1.8 \mathrm{~cm})$, density estimates decreased across all $r$ 's. Density estimates from the 3.6-cm sampling width were statistically different (Tukey means comparisons, $\alpha=0.05$ ) from both the $1.2-\mathrm{cm}$ and $1.8-\mathrm{cm}$ sampling configurations across all $r$ 's.

Density estimate biases varied widely by sampling configuration, with relative biases ranging from $8 \%$ to $28 \%$. Biases increased with increasing sample width, while biases tended to decrease with increasing $r$. This effect was particularly pronounced in the $3.6-\mathrm{cm}$ sampling configuration where the influence of $r$ was the greatest.

Bootstrapped density estimates were very consistent with the sample estimates, reinforcing the veracity of the sample parameters. Bootstrapped estimates showed the same qualitative and quantitative patterns with sample width and $r$ as the non-bootstrapped estimates. Additionally, the bootstrapped relative bias $\left(\mathrm{RB}_{\mathrm{b}}\right)$ showed very similar results to the biases from the original

\begin{tabular}{|c|c|c|c|c|c|c|c|c|c|c|c|c|}
\hline \multirow{2}{*}{$\begin{array}{l}\text { Sample width } \\
(\mathrm{cm}) \dagger\end{array}$} & \multicolumn{3}{|c|}{ Density $(\bar{D})$} & \multicolumn{3}{|c|}{$\begin{array}{c}\text { Relative } \\
\text { bias (RB; \%) }\end{array}$} & \multicolumn{3}{|c|}{$\begin{array}{l}\text { Bootstrap } \\
\text { density }\left(\bar{D}_{\mathrm{b}}\right)\end{array}$} & \multicolumn{3}{|c|}{$\begin{array}{l}\text { Bootstrap standard } \\
\text { error }\left(\mathrm{SE}_{\mathrm{b}}\right)\end{array}$} \\
\hline & $r=4$ & $r=5$ & $r=6$ & $r=4$ & $r=5$ & $r=6$ & $r=4$ & $r=5$ & $r=6$ & $r=4$ & $r=5$ & $r=6$ \\
\hline $1.2^{\mathrm{A}}$ & 2033 & 2019 & 2004 & 10.5 & 9.7 & 8.9 & 2036 & 2017 & 2010 & 167 & 168 & 121 \\
\hline $1.8^{\mathrm{A}}$ & 2101 & 2063 & 2017 & 14.2 & 12.1 & 9.6 & 2104 & 2057 & 2021 & 103 & 209 & 179 \\
\hline $3.6^{\mathrm{B}}$ & $1324^{\mathrm{a}}$ & $1426^{\mathrm{a}}$ & $1528^{b}$ & -28.0 & -22.4 & -16.9 & 1324 & 1428 & 1528 & 54 & 49 & 56 \\
\hline
\end{tabular}

TABLE 2. Summary of measured and bootstrapped density estimates for different sampling-width configurations.

Notes: Actual density was 1840 objects $/ \mathrm{m}^{2}$. See Methods for further detail. Distance measures were recorded from a randomly located point to the $r$ th closest object within a given sample transect.

$\dagger$ Different letters represent statistically different measured and bootstrapped density groups across all sampling configurations (Tukey means comparisons, $\alpha=0.05$ ). 
sample, and consequently, those values were not included in Table 2.

\section{DisCUSSION}

VAT sampling has been shown to be a robust density estimator by Engeman et al. (1994) and Engeman and Sugihara (1998). These studies were extensive simulation studies that assessed the role of spatial distribution, $n$, and $r$ on VAT performance. In contrast, we focused our efforts on the influence of sample width in a population with a nonrandom spatial distribution.

Our results suggest that transect width affects the performance and bias of the VAT sampling technique. As the transect width increases beyond the size of the largest object, density estimates decrease substantially. To our knowledge, no previous studies have identified this behavior. Engeman et al. (1998) made no mention of the width of the VAT used in their study, given that the "objects" in their simulations were dimensionless points and varied only by location. More recently, Engeman et al. (2002) and Engeman and Sterner (2002) used VAT to estimate row crop damage due to herbivory. The behavior of decreasing density estimates with increasing sample width was not noted in their analysis, although an examination of their methods and results shows a congruent pattern.

The formulation of the VAT estimator provides a basis for examining the influence of transect width on the estimator's performance. The probability of encountering $r$ individuals in a transect is a function of the area searched and the density and spatial distribution of organisms within the population. At narrow transect widths, the length searched for the $r$ th individual will be greater than that for wide transect widths, as demonstrated in the results. Consequently, if we expect the estimator to be insensitive to transect width, the product in the denominator of Eq. 1 (which represents the area of the VAT transect) should remain constant across sample widths due to the inverse relationship between the width of the VAT transect and the search length to the $r$ th organism. Put another way, given a specific population density, the area searched until encountering the $r$ th individual will remain constant if the estimator is insensitive to transect width. Our results do not support this conclusion, but instead show that the search length does not decrease proportionally to increases in transect width. For example, a doubling in transect width between $1.8 \mathrm{~cm}$ and $3.6 \mathrm{~cm}$ did not result in a halving of the search distances (Table 1).

The cause of this behavior is not entirely clear. We suspect that its causes are related to the formulation of the density estimator, violations from assumptions of randomly distributed objects, and constraints on their spatial positions. We posit that the spatial constraint of nonoverlapping object positions results in an underestimation of object density. For a fixed area, a population with no constraints on object positions can support higher densities than one with a nonoverlap constraint. As the width of the VAT transect becomes increasingly small (a line), then the probability of the transect intersecting the center of $r$ organisms becomes increasingly small. In this case, the distance traveled to the $r$ th organism is large and the potential influence of the spatial constraint is likely to be reduced. In cases where VAT transect widths are large in relation to the object sizes, and the objects are clumped, the effect of the spatial constraint is likely to be maximized. Of course, in real populations, objects cannot occupy the same place in space. Consequently, these results have distinct implications on the use of the VAT in real field settings. Despite these issues, VAT sampling using narrow transects was shown to be robust with most estimates having relative biases $<10 \%$.

Given its ease of use, we feel an analysis of the practical applications of the VAT is valuable. We suggest the use of transects that are as narrow as possible, but not so narrow as to result in prohibitively long transects and search times. The distance traversed for a given VAT transect width is a function of the density of the organisms and their spatial distribution. In dense populations, the $r$ th individual will be encountered more quickly, even with a narrow transect width. In this case, an obvious consideration is that the transect should be narrow enough to easily determine if an organism lies within the transect boundaries. This can be accomplished by carrying a rod of a given length as described by Parker (1979) or with the aid of an assistant in the field. However, results from the study also show that, as VAT transect width decreases, the variance in the distance measures increases as well. Therefore, narrow transects, particularly in clumped populations, should include more samples $(n)$ in order to improve the precision of the density estimate. When density is low, the transect should not be so narrow that the distance traversed between organisms is prohibitively long. Under these circumstances, wider transects should be used.

In addition to the potential issues of bias, narrow transect widths reduce the amount of searching for the $r$ th individual. As the width of the transect increases, locating the $r$ th organism becomes more difficult, increasing the potential for measurement error as well as the search time. Moreover, if ancillary information is going to be collected, such as organism composition or size, then wide transects will require measurements to be made off of the transect center line. Thus, the narrower the transect, the more the search path resembles a straight line.

The success of a sampling strategy is dependent upon its accuracy and precision, as well as its ease of implementation under field conditions. This is particularly relevant when considering PDEs in that they were 
developed in order to increase the efficiency and speed of sampling. Our results show that VAT sample bias can bs minimized using transect widths that are of similar width (or smaller) to the objects being sampled. Given the ease of use of VAT sampling, further studies should involve VAT sampling within the field in order to compare its implementation with quadrat sampling. Although both quadrat and VAT sampling methods allow for the collection of ancillary data such as size and composition of organisms, the collection of extensive amounts of ancillary data is better fitted to quadrat methods. Quadrat sampling allows for more intensive measurements over a smaller area. These data are pertinent to studies of community dynamics over time. In contrast, VAT sampling, because of its potential speed, allows for less intensive sampling over a larger area. It may prove to be an efficient sampling strategy for studies in which a coarse-grained inventory of a community is required. Potential applications include vegetation mapping or ground truthing of remotely sensed data. Ultimately, the choice of sampling type should be made with regards to the objective of the study in question.

\section{ACKNOWLEDGMENTS}

We thank Michael Barbour for providing the population sampler and for his comments on the manuscript.

\section{Literature Cited}

Clark, P. J., and F. C. Evans. 1954. Distance to nearest neighbor as a measure of spatial relationships in populations. Ecology 35:445-453.

$\rightarrow$ Cottam, G., and J. T. Curtis. 1956. The use of distance measures in phytosociological sampling. Ecology 37:451-460.

Dale, M. T. 1999. Spatial pattern analysis in plant ecology. Cambridge University Press. Cambridge, UK.

Diggle, P. J. 1975. Robust density estimation using distance methods. Biometrika 62:39-48.

Engeman, R. M., B. K. Maedke, and S. F. Beckerman. 2002. Estimating deer damage losses in cabbage. International Biodeterioration and Biodegradation 49:205-207.

Engeman, R. M., and R. T. Sterner. 2002. A comparison of potential labor-saving sampling methods for assessing large mammal damage in corn. Crop Protection 21:101-105.

Engeman, R. M., and R. T. Sugihara. 1998. Optimization of variable area transect sampling using monte carlo simulation. Ecology 79:1425-1434.

$\rightarrow$ Engeman, R. M., R. T. Sugihara, L. F. Pank, and W. E. Dusenberry. 1994. A comparison of plotless density estimators using Monte Carlo simulation. Ecology 75:1769-1779.

Kershaw, K. A. 1973. Quantitative and dynamic plant ecology. Elsevier, New York, New York, USA.

$\rightarrow$ Parker, K. R. 1979. Density estimation by variable area transect. Journal of Wildlife Management 43:484-492.

Schultz, A. M., R. P. Gibbens, and L. Debano. 1961. Artificial populations for teaching and testing range techniques. Journal of Range Management 14:237-242. 